\title{
GLOBAL ATTRACTIVITY OF N-SPECIES COMPETITION SYSTEMS WITH PURE DISTRIBUTED DELAYS
}

\author{
AHMADJAN MUHAMMADHAJI ${ }^{1,2}$ \\ ${ }^{1}$ College of Mathematics and Systems Science, Xinjiang University, Urumqi 830046, China \\ ${ }^{2}$ Institute of Mathematical Physics, Xinjiang University, Urumqi 830046, China
}

Abstract. This paper deals with the general $n$-species Lotka-Volterra competition systems with pure distributed time delays. Based on the construction of the multiple Lyapunov functional, some new sufficient conditions on the global attractivity of the system are established.

Keywords. Competition system; Distributed time delay; Lyapunov functional; Global attractivity.

2010 Mathematics Subject Classification. 34D05, 37B55, 37N25

\section{INTRODUCTION AND PRELIMINARIES}

In this paper, we investigate the following general n-species Lotka-Volterra competitive systems with pure distributed time delays

$$
\dot{x}_{i}(t)=x_{i}(t)\left[r_{i}(t)-\sum_{j=1}^{n} \sum_{l=1}^{m} a_{i j l}(t) \int_{-\tau_{i j l}}^{0} k_{i j l}(s) x_{j}(t+s) d s\right], \quad i=1,2, \cdots, n,
$$

where $x_{i}(t)(i=1,2, \cdots, n)$ represent the density of $n$ competitive species $x_{i}(i=1,2)$ at time $t$, respectively, $\tau_{i j l} \geq 0(i, j=1,2,, \cdots, n, l=1,2, \cdots, m)$ are constants and $\tau_{i j l}(i, j=1,2,, \cdots, n, l=1,2, \cdots, m)$ maybe $+\infty$. Throughout this paper, for system (1.1), we introduce the following hypotheses.

(H1) Functions $r_{i}(t)(i=1,2, \cdots, n)$ are bounded continuous defined on $R_{+}=[0,+\infty]$, and $a_{i j l}(t)$ $(i, j=1,2, \cdots, n, l=1,2, \cdots, m)$ are bounded continuous and nonnegative on $R_{+}$. Delay kernels $k_{i j l}(s)$ $(i, j=1,2, \cdots, n, l=1,2, \cdots, m)$ are nonnegative integrable functions on $\left[-\tau_{i j l}, 0\right]$ satisfying $\int_{-\tau_{i j l}}^{0} k_{i j l}(s) d s$ $=1$.

(H2) For each $(i=1,2, \cdots, n, l=1,2, \cdots, m)$, there exist constants $\eta_{i}>0$, and $\lambda_{i l}>0$ such that

$$
\liminf _{t \rightarrow \infty} \int_{t}^{t+\eta_{i}} r_{i}(s) d s>0, \quad \liminf _{t \rightarrow \infty} \int_{t}^{t+\lambda_{i l}} a_{i i l}(s) d s>0 .
$$

E-mail address: ahmatjanam@aliyun.com.

Received December 26, 2017; Accepted June 4, 2018.

(C) 2018 Journal of Nonlinear Functional Analysis 
In this paper, for system (1.1) we consider the solution with the following initial condition

$$
x_{i}(t)=\phi_{i}(t) \quad \forall t \in[-\tau, 0], i=1,2, \cdots, n,
$$

where $\phi_{i}(t)(i=1,2, \cdots, n)$ are nonnegative continuous functions defined on $[-\tau, 0]$ satisfying $\phi_{i}(0)>$ $0(i=1,2)$ and $\tau=\max \left\{\tau_{i j l}, i, j=1,2, \cdots, n, l=1,2, \cdots, m\right\}$.

Both with the variable time delays and distributed time delays of system (1.1) were discussed in [1]. In 2015, Muhammadhaji Mahemuti and Teng [1] established the sufficient conditions on the existence of periodic solutions for system (1.1) by using the Mawhin's continuation theorem. In the theory of mathematical biology, system (1.1) is very important mathematical model which describe multiple-time $n$-species interactional population dynamics in a non-autonomous environment. As we known, one of the most famous models for dynamics of population is the Lotka-Volterra competition system. In recent years, population dynamical competition systems have been studied extensively (see [1]-[14] and the references therein) and some of them are described as autonomous or non-autonomous non-delayed differential equations; see [2]-[6] and the references therein. But most of them are on the dynamics of population Lotka-Volterra competition systems with delays, which are useful for the control of the population of mankind, animals and the environment [7]-[14]. For example, Lv, Yan and Lu [7] considered the following competitor-competitor-mutualist Lotka-Volterra systems with pure delays

$$
\begin{aligned}
\dot{x}_{1}(t)= & x_{1}(t)\left(r_{1}(t)-a_{11}(t) x_{1}\left(t-\tau_{11}(t)\right)\right. \\
& \left.-a_{12}(t) x_{2}\left(t-\tau_{12}(t)\right)+a_{13}(t) x_{3}\left(t-\tau_{13}(t)\right)\right), \\
\dot{x}_{2}(t)= & x_{2}(t)\left(r_{2}(t)-a_{21}(t) x_{1}\left(t-\tau_{21}(t)\right)\right. \\
& \left.-a_{22}(t) x_{2}\left(t-\tau_{22}(t)\right)+a_{23}(t) x_{3}\left(t-\tau_{23}(t)\right)\right), \\
\dot{x}_{3}(t)= & x_{3}(t)\left(r_{3}(t)+a_{31}(t) x_{1}\left(t-\tau_{31}(t)\right)\right. \\
& \left.+a_{32}(t) x_{2}\left(t-\tau_{32}(t)\right)-a_{33}(t) x_{3}\left(t-\tau_{33}(t)\right)\right) .
\end{aligned}
$$

They obtained sufficient conditions on the existence and global attractivity of positive periodic solution for system (1.2) by means of the Krasnosselsii's fixed point theorem and the construction of the Lyapunov functional. In 1999, Fan, Wang and Jiang [9] studied the following non-autonomous $n$-species LotkaVolterra competitive systems with pure continuous time delays

$$
\dot{y}(t)=y(t)\left[r_{i}(t)-\sum_{j=1}^{n} a_{i j}(t) y_{j}\left(t-\tau_{i j}(t)\right)\right], \quad i=1,2, \cdots, n .
$$

By using the method of the coincidence degree and the Lyapunov functional, some sufficient conditions on the existence and global attractivity of positive periodic solutions are established for system (1.3). In 2011, Yan and Liu [12] studied the following non-autonomous $n$-species Lotka-Volterra competitive systems with feedback controls and multiple time delays

$$
\begin{aligned}
\dot{y}_{i}(t)= & b_{i}\left(y_{i}(t)\right)\left[r_{i}(t)-\sum_{j=1}^{n} a_{i j}(t) y_{j}(t)-\sum_{j=1}^{n} \sum_{k=1}^{m} b_{i j k}(t) y_{j}\left(t-\tau_{i j k}(t)\right)\right. \\
& \left.-\sum_{j=1}^{n} \sum_{k=1}^{m} \int_{-\infty}^{t} c_{i j k}(t, \tau) y_{j}(\tau) d \tau-d_{i}(t) u_{i}(t)-e_{i}(t) u_{i}\left(t-\sigma_{i}(t)\right)\right], \\
\dot{u}_{i}(t)= & -\alpha_{i}(t) u_{i}(t)+\beta_{i}(t) x_{i}(t)+\gamma_{i}(t) x_{i}\left(t-\delta_{i}(t)\right), \quad i=1,2, \cdots, n .
\end{aligned}
$$

By employing the fixed point index theory on cones, the necessary and sufficient condition for the global existence of the positive periodic solution of the systems is obtained for system (1.4), and by constructing a suitable Lyapunov functional, the sufficient conditions for the global asymptotic stability of the positive 
periodic solution of the system are given. Systems (1.2) and (1.3) are pure delayed systems, however, the authors have not considered the global attractivity of the system for pure delay case. As far as we know, studies on the global attractivity of Lotka-Volterra systems with pure delays have not been fully investigated. In this paper, we study system (1.1). Based on the construction of the multiple Lyapunov functional, we establish some new sufficient conditions which guarantee the pure delayed system is globally attractive. Our results improved the corresponding results in [1].

Lemma 1.1. Set $R_{+}^{n}=\left\{\left(x_{1}, x_{2}, \cdots, x_{n}(t)\right): x_{i}>0, i=1,2, \cdots, n\right\}$ is positively invariant for system (1.1).

We consider the following nonautonomous logistic equation

$$
\frac{d x(t)}{d t}=x(t)(a(t)-b(t) x(t)),
$$

where the functions $a(t)$ and $b(t)$ are bounded continuous on $R_{+}$.

Lemma 1.2. [6] Suppose that there are constants $\eta>0, \lambda>0$ such that

$$
\liminf _{t \rightarrow \infty} \int_{t}^{t+\eta} a(s) d s>0, \quad \liminf _{t \rightarrow \infty} \int_{t}^{t+\lambda} b(s) d s>0 .
$$

Then, there exist constants $M>m>0$ such that

$$
m \leq \liminf _{t \rightarrow \infty} x(t) \leq \lim _{t \rightarrow \infty} \sup x(t) \leq M
$$

for any positive solution $x(t)$ of Eq. (1.5), where $M=\lim _{t \rightarrow \infty} \sup \frac{a(t)}{b(t)}$

\section{MAIN RESULTS}

Theorem 2.1. If assumptions $(\boldsymbol{H 1})$ and $(\boldsymbol{H} 2)$ hold, then there exist positive constants $M_{i}(i=1,2, \cdots, n)$ such that

$$
\lim _{t \rightarrow \infty} \sup x_{i}(t) \leq M_{i}
$$

for any positive solution $x_{i}(t)$ of system (1.1)

Proof. Let $\left(x_{1}(t), x_{2}(t), \cdots, x_{n}(t)\right)$ be a solution of system (1.1). For each $i \in\{1,2, \cdots, n\}$, we have

$$
\begin{aligned}
\frac{d x_{i}(t)}{d t} & =x_{i}(t)\left[r_{i}(t)-\sum_{j=1}^{n} \sum_{l=1}^{m} a_{i j l}(t) \int_{-\tau_{i j l}}^{0} k_{i j l}(s) x_{j}(t+s) d s\right] \\
& \leq r_{i}(t) x_{i}(t) \quad \text { for } t \geq 0 .
\end{aligned}
$$

Hence, for any $t \geq 0, \theta \leq 0$ and $t+\theta>0$, integrating from $t+\theta$ to $t$ on both sides of (2.1) produces

$$
x_{i}(t+\theta) \geq x_{i}(t) \exp \int_{t}^{t+\theta} r_{i}(s) d s .
$$

Therefore, for any $t \geq \tau$, from (2.2) and the ith equation of system (1.4), we have

$$
\begin{aligned}
\frac{d x_{i}(t)}{d t} & \leq x_{i}(t)\left[r_{i}(t)-\sum_{l=1}^{m} a_{i i l}(t) \int_{-\tau_{i l}}^{0} k_{i i l}(s) x_{i}(t+s) d s\right] \\
& \leq x_{i}(t)\left(r_{i}(t)-\sum_{l=1}^{m} a_{i i l}(t) \exp \int_{t}^{t+s} r_{i}(\theta) d \theta x_{i}(t)\right) .
\end{aligned}
$$

Since, for any $t \geq \tau$ and $s \in[-\tau, 0]$,

$$
\int_{t}^{t+s} r_{i}(\theta) d \theta \geq-\tau r_{i}^{M}
$$


we have

$$
\left.\frac{d x_{i}(t)}{d t} \leq x_{i}(t)\left[r_{i}(t)-b_{i} \sum_{l=1}^{m} a_{i i l}(t) x_{i}(t)\right)\right]
$$

for $t \geq \tau$, where $b_{i}=\exp \left(-\tau r_{i}^{M}\right)$ and $r_{i}^{M}=\sup _{t \in[0,+\infty)}\left\{r_{i}(t)\right\}$. Letting

$$
a_{i}(t)=b_{i} \sum_{l=1}^{m} a_{i i l}(t)
$$

we consider the following auxiliary equation

$$
\frac{d y_{i}(t)}{d t}=y_{i}(t)\left(r_{i}(t)-a_{i}(t) y_{i}(t)\right) .
$$

From condition (H2) and Lemma 1.2, there exist constants $\bar{x}_{i}>0$ such that

$$
\lim _{t \rightarrow \infty} \sup y_{i}(t)<\bar{x}_{i} .
$$

for any positive solution $y_{i}(t)$ of Eq. (2.3). Let $y_{i}^{*}(t)$ be solution of Eq. (2.3) with initial conditions $y_{i}^{*}(\tau)=x_{i}(\tau)$. In view of the comparison theorem, we have

$$
x_{i}(t)<y_{i}^{*}(t) \text { for } t \geq \tau .
$$

Finally, we have

$$
\lim _{t \rightarrow \infty} \sup x_{i}(t)<\bar{x}_{i}=: M_{i} .
$$

This completes the proof of this Theorem.

The following result is on the global attractivity of system (1.1).

Theorem 2.2. Assume that assumptions $(\boldsymbol{H 1})$ and $(\boldsymbol{H} 2)$ hold. Further, if the following assumption $\left(\mathrm{H}_{3}\right)$ holds

(H3) There exist constants $\mu_{i}>0(i=1,2, \cdots, n)$ such that

$$
\liminf _{t \rightarrow \infty} L_{i}(t)>0, \quad i=1,2, \cdots, n,
$$

where

$$
\begin{aligned}
L_{i}(t)= & \sum_{l=1}^{m} \mu_{i} a_{i i l}(t)-\sum_{i \neq j, j=1}^{n} \sum_{l=1}^{m} \mu_{j} \int_{-\tau_{j i l}}^{0} k_{j i l}(s) a_{j i l}(t-s) d s \\
& -\sum_{l=1}^{m} \mu_{i} \int_{-\tau_{i l l}}^{0} k_{i i l}(s) \int_{t}^{t-s} a_{i i l}(\eta) d \eta d s\left[r_{i}(t)+M_{j} \sum_{j=1}^{n} \sum_{l=1}^{m} a_{i j l}(t)\right] \\
& -\mu_{j} M_{j} \sum_{l=1}^{m} \int_{-\tau_{j j l}}^{0} k_{j j l}(s) \int_{t-s}^{t-2 s} a_{j j l}(\eta) d \eta d s\left[\sum_{j=1}^{n} \sum_{l=1}^{m} \int_{-\tau_{j i l}}^{0} k_{j i l}(s) a_{j i l}(t-s) d s\right],
\end{aligned}
$$

then system (1.1) is globally attractive.

Proof. Assume that $x^{*}(t)=\left(x_{1}^{*}(t), x_{2}^{*}(t), \cdots, x_{n}^{*}(t)\right)$ and $x(t)=\left(x_{1}(t), x_{2}(t), \cdots, x_{n}(t)\right)$ are any two positive solutions of system (1.1). From Theorem 2.1, there exist positive constants $T$ and $M_{i}(i=1,2, \cdots, n)$ such that, for all $t>T$,

$$
0<x_{i}^{*}(t), x(t) \leq M_{i}, \quad i=1,2, \cdots, n .
$$

Let

$$
V_{i 1}(t)=\left|\ln x_{i}^{*}(t)-\ln x_{i}(t)\right|, \quad i=1,2, \cdots, n .
$$


Calculating the upper right derivation of $V_{i 1}(t)$ along system (1.1) for $i=1,2, \cdots, n$, we have

$$
\begin{aligned}
& D^{+} V_{i 1}(t)=\operatorname{sign}\left(x_{i}^{*}(t)-x_{i}(t)\right)\left[-\sum_{l=1}^{m} a_{i i l}(t) \int_{-\tau_{i j l}}^{0} k_{i i l}(s)\left(x_{j}^{*}(t+s)-x_{j}(t+s)\right) d s\right. \\
& \left.-\sum_{i \neq j, j=1}^{n} \sum_{l=1}^{m} a_{i j l}(t) \int_{-\tau_{i j l}}^{0} k_{i j l}(s)\left(x_{j}^{*}(t+s)-x_{j}(t+s)\right) d s\right] \\
& =\operatorname{sign}\left(x_{i}^{*}(t)-x_{i}(t)\right)\left[-\sum_{l=1}^{m} a_{i i l}(t)\left(x_{i}^{*}(t)-x_{i}(t)\right)+\sum_{l=1}^{m} a_{i i l}(t) \int_{-\tau_{i i l}}^{0} k_{i i l}(s)\right. \\
& \left.\times \int_{t+s}^{t}\left(\dot{x}_{i}^{*}(u)-\dot{x}_{i}(u)\right) d u d s-\sum_{i \neq j, j=1}^{n} \sum_{l=1}^{m} a_{i j l}(t) \int_{-\tau_{i j l}}^{0} k_{i j l}(s)\left(x_{j}^{*}(t+s)-x_{j}(t+s)\right) d s\right] \\
& =\operatorname{sign}\left(x_{i}^{*}(t)-x_{i}(t)\right)\left[-\sum_{l=1}^{m} a_{i i l}(t)\left(x_{i}^{*}(t)-x_{i}(t)\right)+\sum_{l=1}^{m} a_{i i l}(t) \int_{-\tau_{i i l}}^{0} k_{i i l}(s)\right. \\
& \times \int_{t+s}^{t}\left(x_{i}^{*}(u)\left[r_{i}(u)-\sum_{j=1}^{n} \sum_{l=1}^{m} a_{i j l}(u) \int_{-\tau_{i j l}}^{0} k_{i j l}(s) x_{j}^{*}(u+s) d s\right]-x_{i}(u)\left[r_{i}(u)\right.\right. \\
& \left.\left.-\sum_{j=1}^{n} \sum_{l=1}^{m} a_{i j l}(u) \int_{-\tau_{i j l}}^{0} k_{i j l}(s) x_{i}(u+s) d s\right]\right) d t d s-\sum_{i \neq j, j=1}^{n} \sum_{l=1}^{m} a_{i j l}(t) \int_{-\tau_{i j l}}^{0} k_{i j l}(s) \\
& \left.\times\left(x_{j}^{*}(t+s)-x_{j}(t+s)\right) d s\right] \\
& =\operatorname{sign}\left(x_{i}^{*}(t)-x_{i}(t)\right)\left[-\sum_{l=1}^{m} a_{i i l}(t)\left(x_{i}^{*}(t)-x_{i}(t)\right)-\sum_{i \neq j, j=1}^{n} \sum_{l=1}^{m} a_{i j l}(t) \int_{-\tau_{i j l}}^{0} k_{i j l}(s)\right. \\
& \times\left(x_{j}^{*}(t+s)-x_{j}(t+s)\right) d s+\sum_{l=1}^{m} a_{i i l}(t) \int_{-\tau_{i l l}}^{0} k_{i i l}(s) \int_{t+s}^{t}\left(\left(x_{i}^{*}(u)-x_{i}(u)\right)\right. \\
& \times\left[r_{i}(u)-\sum_{j=1}^{n} \sum_{l=1}^{m} a_{i j l}(u) \int_{-\tau_{i j l}}^{0} k_{i j l}(s) x_{j}(u+s) d s\right]+x_{i}^{*}(u)\left[-\sum_{j=1}^{n} \sum_{l=1}^{m} a_{i j l}(u)\right. \\
& \left.\left.\left.\times \int_{-\tau_{i j l}}^{0} k_{i j l}(s)\left(x_{j}^{*}(u+s)-x_{j}(u+s)\right) d s\right]\right) d u d s\right] \\
& \leq-\sum_{l=1}^{m} a_{i i l}(t)\left|x_{i}^{*}(t)-x_{i}(t)\right|+\sum_{i \neq j, j=1}^{n} \sum_{l=1}^{m} a_{i j l}(t) \int_{-\tau_{i j l}}^{0} k_{i j l}(s) \\
& \times\left|x_{j}^{*}(t+s)-x_{j}(t+s)\right| d s+\sum_{l=1}^{m} a_{i i l}(t) \int_{-\tau_{i i l}}^{0} k_{i i l}(s) \int_{t+s}^{t}\left(\left|x_{i}^{*}(u)-x_{i}(u)\right|\right. \\
& \times\left[r_{i}(u)+\sum_{j=1}^{n} \sum_{l=1}^{m} a_{i j l}(u) \int_{-\tau_{i j l}}^{0} k_{i j l}(s) x_{j}(u+s) d s\right]+x_{i}^{*}(u)\left[\sum_{j=1}^{n} \sum_{l=1}^{m} a_{i j l}(u)\right. \\
& \left.\left.\times \int_{-\tau_{i j l}}^{0} k_{i j l}(s)\left|x_{j}^{*}(u+s)-x_{j}(u+s)\right| d s\right]\right) d u d s .
\end{aligned}
$$

Define

$$
V_{i 2}(t)=\sum_{i \neq j, j=1}^{n} \sum_{l=1}^{m} \int_{-\tau_{i j l}}^{0} k_{i j l}(s) \int_{t+s}^{t} a_{i j l}(\theta-s)\left|x_{j}^{*}(\theta)-x_{j}(\theta)\right| d \theta d s
$$




$$
\begin{aligned}
& +\sum_{l=1}^{m} \int_{-\tau_{i i l}}^{0} k_{i i l}(s) \int_{t}^{t-s} \int_{\eta+s}^{t} a_{i i l}(\eta)\left(\left|x_{i}^{*}(u)-x_{i}(u)\right|\right. \\
& \times\left[r_{i}(u)+\sum_{j=1}^{n} \sum_{l=1}^{m} a_{i j l}(u) \int_{-\tau_{i j l}}^{0} k_{i j l}(s) x_{j}(u+s) d s\right]+x_{i}^{*}(u)\left[\sum_{j=1}^{n} \sum_{l=1}^{m} a_{i j l}(u)\right. \\
& \left.\left.\times \int_{-\tau_{i j l}}^{0} k_{i j l}(s)\left|x_{j}^{*}(u+s)-x_{j}(u+s)\right| d s\right]\right) d u d \eta d s, \quad i=1,2, \cdots, n .
\end{aligned}
$$

Calculating the upper right derivative of $V_{i 1}(t)+V_{i 2}(t)$, we find from (2.5) that

$$
\begin{aligned}
V_{i 1}(t)+V_{i 2}(t) \leq & -\sum_{l=1}^{m} a_{i i l}(t)\left|x_{i}^{*}(t)-x_{i}(t)\right|+\sum_{i \neq j, j=1}^{n} \sum_{l=1}^{m} \int_{-\tau_{i j l}}^{0} k_{i j l}(s) a_{i j l}(t-s) d s \\
& \times\left|x_{j}^{*}(t)-x_{j}(t)\right|+\sum_{l=1}^{m} \int_{-\tau_{i i l}}^{0} k_{i i l}(s) \int_{t}^{t-s} a_{i i l}(\eta)\left(\left|x_{i}^{*}(t)-x_{i}(t)\right|\right. \\
& \times\left[r_{i}(t)+\sum_{j=1}^{n} \sum_{l=1}^{m} a_{i j l}(t) \int_{-\tau_{i j l}}^{0} k_{i j l}(s) x_{j}(t+s) d s\right]+x_{i}^{*}(t)\left[\sum_{j=1}^{n} \sum_{l=1}^{m} a_{i j l}(t)\right. \\
& \left.\left.\times \int_{-\tau_{i j l}}^{0} k_{i j l}(s)\left|x_{j}^{*}(t+s)-x_{j}(t+s)\right| d s\right]\right) d \eta d s \\
\leq & -\sum_{l=1}^{m} a_{i i l}(t)\left|x_{i}^{*}(t)-x_{i}(t)\right|+\sum_{i \neq j, j=1}^{n} \sum_{l=1}^{m} \int_{-\tau_{i j l}}^{0} k_{i j l}(s) a_{i j l}(t-s) d s \\
& \times\left|x_{j}^{*}(t)-x_{j}(t)\right|+\sum_{l=1}^{m} \int_{-\tau_{i i l}}^{0} k_{i i l}(s) \int_{t}^{t-s} a_{i i l}(\eta) d \eta d s\left[r_{i}(t)+M_{j} \sum_{j=1}^{n} \sum_{l=1}^{m} a_{i j l}(t)\right] \\
& \times\left|x_{i}^{*}(t)-x_{i}(t)\right|+M_{i} \sum_{l=1}^{m} \int_{-\tau_{i l l}}^{0} k_{i i l}(s) \int_{t}^{t-s} a_{i i l}(\eta) d \eta d s\left[\sum_{j=1}^{n} \sum_{l=1}^{m} a_{i j l}(t)\right. \\
& \left.\times \int_{-\tau_{i j l}}^{0} k_{i j l}(s)\left|x_{j}^{*}(t+s)-x_{j}(t+s)\right| d s\right] .
\end{aligned}
$$

Define

$$
V_{i}(t)=V_{i 1}(t)+V_{i 2}(t)+V_{i 3}(t)
$$

where

$$
\begin{aligned}
V_{i 3}(t)= & M_{i} \sum_{l=1}^{m} \int_{-\tau_{i i l}}^{0} k_{i i l}(s) \int_{t+s}^{t}\left(\int_{u-s}^{u-2 s} a_{i i l}(\eta) d \eta\right)\left[\sum_{j=1}^{n} \sum_{l=1}^{m} a_{i j l}(t)\right. \\
& \left.\times \int_{-\tau_{i j l}}^{0} k_{i j l}(s) a_{i j l}(u-s)\left|x_{j}^{*}(u)-x_{j}(u)\right| d s\right] d u d s, \quad i=1,2, \cdots, n .
\end{aligned}
$$

Calculating the upper right derivative of $V_{i}(t)$, we find from (2.6) that 


$$
\begin{aligned}
V_{i}(t) \leq & \sum_{l=1}^{m} a_{i i l}(t)\left|x_{i}^{*}(t)-x_{i}(t)\right|+\sum_{i \neq j, j=1}^{n} \sum_{l=1}^{m} \int_{-\tau_{i j l}}^{0} k_{i j l}(s) a_{i j l}(t-s) d s \\
& \times\left|x_{j}^{*}(t)-x_{j}(t)\right|+\sum_{l=1}^{m} \int_{-\tau_{i i l}}^{0} k_{i i l}(s) \int_{t}^{t-s} a_{i i l}(\eta) d \eta d s\left[r_{i}(t)+M_{j} \sum_{j=1}^{n} \sum_{l=1}^{m} a_{i j l}(t)\right] \\
& \times\left|x_{i}^{*}(t)-x_{i}(t)\right|+M_{i} \sum_{l=1}^{m} \int_{-\tau_{i i l}}^{0} k_{i i l}(s) \int_{t-s}^{t-2 s} a_{i i l}(\eta) d \eta d s\left[\sum_{j=1}^{n} \sum_{l=1}^{m} a_{i j l}(t)\right. \\
& \left.\times \int_{-\tau_{i j l}}^{0} k_{i j l}(s) a_{i j l}(t-s) d s\right]\left|x_{j}^{*}(t)-x_{j}(t)\right| .
\end{aligned}
$$

Finally, we define a Lyapunov function as follows

$$
V(t)=\sum_{i=1}^{n} \mu_{i} V_{i}(t)
$$

Calculating the upper right derivation of $V(t)$, we find from (2.7) that

$$
D^{+} V(t) \leq-\sum_{i=1}^{n} L_{i}(t)\left|x_{i}^{*}(t)-x_{i}(t)\right| \quad \forall t \geq T
$$

where

$$
\begin{aligned}
L_{i}(t)= & \sum_{l=1}^{m} \mu_{i} a_{i i l}(t)-\sum_{i \neq j, j=1}^{n} \sum_{l=1}^{m} \mu_{j} \int_{-\tau_{j i l}}^{0} k_{j i l}(s) a_{j i l}(t-s) d s \\
& -\sum_{l=1}^{m} \mu_{i} \int_{-\tau_{i l l}}^{0} k_{i i l}(s) \int_{t}^{t-s} a_{i i l}(\eta) d \eta d s\left[r_{i}(t)+M_{j} \sum_{j=1}^{n} \sum_{l=1}^{m} a_{i j l}(t)\right] \\
& -\mu_{j} M_{j} \sum_{l=1}^{m} \int_{-\tau_{j j l}}^{0} k_{j j l}(s) \int_{t-s}^{t-2 s} a_{j j l}(\eta) d \eta d s\left[\sum_{j=1}^{n} \sum_{l=1}^{m} \int_{-\tau_{j i l}}^{0} k_{j i l}(s) a_{j i l}(t-s) d s\right] .
\end{aligned}
$$

From assumption (H3), there exist constants $L>0$ and $T_{0}>T$ such that, for all $t>T_{0}$,

$$
L_{i}(t) \geq L>0, \quad i=1,2, \cdots, n .
$$

Integrating from $T_{0}$ to $t$ on both sides of (2.8), we find from (2.9) that

$$
V(t)+\int_{T_{0}}^{t}\left(\sum_{i=1}^{n} L\left|x_{i}^{*}(s)-x_{i}(s)\right|\right) d s \leq V\left(T_{0}\right), \quad t \geq T_{0} .
$$

It follows that

$$
\int_{0}^{t}\left(\sum_{i=1}^{n}\left|x_{i}^{*}(s)-x_{i}(s)\right|\right) d s \leq \frac{V\left(T_{0}\right)}{L}, \quad t \geq 0 .
$$

Hence, $V(t)$ is bounded on $\left[T_{0},+\infty\right)$. Then

$$
\int_{T_{0}}^{t}\left(\sum_{i=1}^{n} L(t)\left|x_{i}^{*}(s)-x_{i}(s)\right|\right) d s<\infty .
$$

From Theorem 2.1, we can obtain that $\left(x_{i}^{*}(t)-x_{i}(t)\right)(i=1,2, \cdots, n)$ and their derivatives are remain bounded on $\left[T_{0},+\infty\right)$. Therefore, $\sum_{i=1}^{n}\left|x_{i}^{*}(t)-x_{i}(t)\right|$ is uniformly continuous on $[0,+\infty)$. From the Barbalats lemma, it follows that

$$
\lim _{t \rightarrow+\infty} \sum_{i=1}^{n}\left|x_{i}^{*}(t)-x_{i}(t)\right|=0 .
$$


Hence,

$$
\lim _{t \rightarrow+\infty}\left(x_{i}^{*}(t)-x_{i}(t)\right)=0, \quad i=1,2, \cdots, n .
$$

This completes the proof of this Theorem.

\section{AN APPLICATION}

In this section, as an application of the main results in this paper, we consider the following nonautonomous single species system with pure distributed delays

$$
\dot{x}(t)=x(t)\left[r(t)-\sum_{i=1}^{2} \sum_{l=1}^{m} a_{i l}(t) \int_{-\tau_{i l}}^{0} k_{i l}(s) x_{i}(t+s) d s\right],
$$

where $x(t)$ represent the density of single species $x$ at time $t$, respectively, $\tau_{i l} \geq 0(i=1,2, l=1,2, \cdots, m)$ is a constant and $\tau_{i l}(i, j=1,2, l=1,2, \cdots, m)$ maybe $+\infty$. For system (3.1), we introduce the following hypotheses.

(H4) Function $r(t)$ is bounded continuous defined on $R_{+}=[0,+\infty]$, and $a_{i l}(t)(i=1,2, l=1,2, \cdots, m)$ are bounded continuous and nonnegative on $R_{+}$. Delay kernels $k_{i l}(s)(i=1,2, l=1,2, \cdots, m)$ are nonnegative integrable functions on $\left[-\tau_{i l}, 0\right]$ satisfying $\int_{-\tau_{i l}}^{0} k_{i l}(s) d s=1$.

(H5) For each $(l=1,2, \cdots, m)$, there exist constants $\eta_{1}>0, \lambda_{1 l}>0$ such that

$$
\liminf _{t \rightarrow \infty} \int_{t}^{t+\eta_{0}} r(s) d s>0, \quad \liminf _{t \rightarrow \infty} \int_{t}^{t+\lambda_{1 l}} a_{1 l}(s) d s>0 .
$$

On the bundedness and global attractivity of system (3.1) we have the following results.

Corollary 3.1. If (H4) and (H5) hold, then there exists a positive constant $M$ such that

$$
\lim _{t \rightarrow \infty} \sup x(t) \leq M
$$

for any positive solution $x(t)$ of system (3.1)

Corollary 3.2. Assume that $(\boldsymbol{H 4})$ and $(\boldsymbol{H 5})$ hold. Further suppose that the following $\left(H_{6}\right)$ holds

(H6) There exist a constant $\mu>0$ such that

$$
\liminf _{t \rightarrow \infty} L(t)>0
$$

where

$$
\begin{aligned}
L(t)= & \sum_{l=1}^{m} \mu a_{1 l}(t)-\sum_{l=1}^{m} \mu \int_{-\tau_{2 l}}^{0} k_{2 l}(s) a_{2 l}(t-s) d s \\
& -\sum_{l=1}^{m} \mu \int_{-\tau_{1 l}}^{0} k_{1 l}(s) \int_{t}^{t-s} a_{1 l}(\eta) d \eta d s\left[r(t)+M \sum_{i=1}^{2} \sum_{l=1}^{m} a_{i l}(t)\right] \\
& -\mu M \sum_{l=1}^{m} \int_{-\tau_{1 l}}^{0} k_{1 l}(s) \int_{t-s}^{t-2 s} a_{1 l}(\eta) d \eta d s\left[\sum_{i=1}^{2} \sum_{l=1}^{m} \int_{-\tau_{i l}}^{0} k_{i l}(s) a_{i l}(t-s) d s\right]
\end{aligned}
$$

Then system (3.1) is globally attractive. 


\section{DisCUSSION}

In this paper, one class of general non-autonomous $n$-species Lotka-Volterra competition systems with pure distributed time delays are proposed and analyzed to study the effect of distributed time delays on the global attractivity of the system. By means of the construction of the multiple Lyapunov functional, we have obtained the sufficient conditions for the global attractivity of the system. The purpose of the construction of the multiple Lyapunov functional is to produce non-delayed terms in the system so that we can control the delayed terms by non-delayed terms. From the conditions of Theorem 2.2 and Corollary 3.2, we can see that the distributed time delays have effect on the global attractivity of the system, and further from conditions (H3) and (H6) we can see that the upper bounds $M_{i}(i=1,2, \cdots, n)$ and $M$ are very useful to construction of the Lyapunov functional.

We have a interesting future work that is to study the following general non-autonomous $n$-species Lotka-Volterra cooperative systems with pure distributed time delays

$$
\begin{aligned}
\dot{x}_{i}(t)= & x_{i}(t)\left[r_{i}(t)-\sum_{l=1}^{m} a_{i i l}(t) \int_{-\tau_{i l l}}^{0} k_{i i l}(s) x_{i}(t+s) d s\right. \\
& \left.+\sum_{i \neq j, j=1}^{n} \sum_{l=1}^{m} a_{i j l}(t) \int_{-\tau_{i j l}}^{0} k_{i j l}(s) x_{j}(t+s) d s\right], \quad i=1,2, \cdots, n,
\end{aligned}
$$

System (4.1) was discussed in [15], the authors in [15] established the sufficient conditions on the existence of periodic solutions for system (4.1) by using the Mawhin's continuation theorem. For the global attractivity of system (4.1), we introduce the following assumption (H7)

(H7) There exist constants $\gamma_{i}>0(i=1,2, \cdots, n)$ such that

$$
\liminf _{t \rightarrow \infty} G_{i}(t)>0, \quad i=1,2, \cdots, n,
$$

where

$$
\begin{aligned}
G_{i}(t)= & \sum_{l=1}^{m} \gamma_{i} a_{i i l}(t)-\sum_{i \neq j, j=1}^{n} \sum_{l=1}^{m} \gamma_{j} \int_{-\tau_{j i l}}^{0} k_{j i l}(s) a_{j i l}(t-s) d s \\
& -\sum_{l=1}^{m} \gamma_{i} \int_{-\tau_{i l l}}^{0} k_{i i l}(s) \int_{t}^{t-s} a_{i i l}(\eta) d \eta d s\left[r_{i}(t)+M_{j}^{*} \sum_{j=1}^{n} \sum_{l=1}^{m} a_{i j l}(t)\right] \\
& -\gamma_{j} M_{j}^{*} \sum_{l=1}^{m} \int_{-\tau_{j j l}}^{0} k_{j j l}(s) \int_{t-s}^{t-2 s} a_{j j l}(\eta) d \eta d s\left[\sum_{j=1}^{n} \sum_{l=1}^{m} \int_{-\tau_{j i l}}^{0} k_{j i l}(s) a_{j i l}(t-s) d s\right]
\end{aligned}
$$

and $M_{i}^{*}(i=1,2, \cdots, n)$ are the upper bounds of solutions $x_{i}(t)(i=1,2, \cdots, n)$ in system (4.1). However, the most difficult part in this study is to find the upper bounds for the solutions $x_{i}(t)(i=1,2, \cdots, n)$.

\section{Acknowledgement}

This work was supported by the National Natural Science Foundations of China under Grant No. 11601464.

\section{REFERENCES}

[1] A. Muhammadhaji, R. Mahemuti, Z. Teng, Periodic solutions for n-species Lotka-Volterra competitive systems with pure delays, China J. Math. 2015 (2015), Article ID 856959.

[2] S. Ahmad, Convergence and ultimate bounds of the nonautonomous Volterra-Lotka competition equation, J. Math. Anal. Appl. 127 (1987), 377-387.

[3] S. Ahmad, On the nonautonomous Volterra-Lotka competition equations, Proc. Amer. Math. Soc. 117 (1993), $199-204$. 
[4] Q.L. Peng, L.S. Chen, Asymptotic behavior of the nonautonomous two-species Lotka-Volterra competition models, Comp. Math. Appl. 27 (1994), 53-60.

[5] G.Z. Zeng, L.S. Chen, J.F. Chen, Persistence and periodic orbits for two-species nonautonomous diffusion Lotka-Volterra models, Mathl. Comput. Modelling. 20 (1994), 69-80.

[6] Z. Teng, Z. Li, Permanence and asymptotic behavior of the n-species nonautonomous Lotka-Volterra competitive systems, Comput. Math. Appl. 39 (2000), 107-116.

[7] X. Lv, P. Yan, S. Lu, Existence and global attractivity of positive periodic solutions of competitor-competitor-mutualist Lotka-Volterra systems with deviating arguments, Math. Comput. Modelling. 51 (2010), 823-832.

[8] H. Fang, Z. Wang, Existence and global attractivity of positive periodic solutions for delay Lotka-Volterra competition patch systems with stocking, J. Math. Anal. Appl. 293 (2004), 190-209.

[9] M. Fan, K. Wang, D. Jiang, Existence and global attractivity of positive periodic solutions of periodic n-species LotkaVolterra competition systems with several deviating arguments, Math. Biosci. 160 (1999), 47-61.

[10] L. Zhang, H. Li, X. Zhang, Periodic solutions of competition Lotka-Volterra dynamic system on time scales, Comput. Math. Appl. 57 (2009), 1204-1211.

[11] F. Wei, Y. Lin, L. Que, Y. Chen, Y. Wu, Y. Xue, Periodic solution and global stability for a nonautonomous competitive Lotka-Volterra diffusion system, Appl. Math. Comput. 216 (2010), 3097-3104.

[12] J. Yan, G. Liu, Periodicity and stability for a Lotka-Volterra type competition system with feedback controls and deviating arguments, Nonlinear Anal. 74 (2011), 2916-2928.

[13] F. Chen, On the periodic solutions of periodic multi-species Kolmogorov type competitive system with delays and feedback controls, Appl. Math. Comput. 180 (2006), 366-373.

[14] C. Egami, Positive periodic solutions of nonautonomous delay competitive systems with weak Allee effect, Nonlinear Anal. 10 (2009), 494-505.

[15] A. Muhammadhaji, Z. Teng, Positive periodic solutions of n-species Lotka-Volterra cooperative systems with delays, Vietnam J. Math. 40 (2012), 453-467. 